\title{
novembre

\section{Imaginer l'avenir des territoires pour relever les défis de la démographie et de l'emploi en Afrique subsaharienne}

Jean-Michel SOURISSEAU - Nathalie BOUGNOUX - Jean-François BÉLIĖRES Robin BOURGEOIS - Mamy SOUMARÉ - Patrick RASOLOFO

En Afrique subsaharienne, l'ampleur du défi démographique impose d'anticiper les objectifs à atteindre à I'horizon de la prochaine génération, et ce dans tous les secteurs d'activité. Nous proposons une méthode opérationnelle que les décideurs et les acteurs des territoires ruraux peuvent mettre en place pour imaginer le futur et élaborer leur stratégie de développement. Cette méthode de prospective territoriale combine un diagnostic des dynamiques locales passées, une projection de la population du territoire à 20 ans et un travail de prospective participative. Elle permet de réfléchir collectivement à des trajectoires soutenables et elle offre un cadre pour suivre et piloter l'action publique territoriale.
La forte croissance démographique des zones rurales a un impact inédit sur les stratégies de développement et sur la gestion des ressources

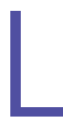

'Afrique au Sud du Sahara suit une trajectoire de développement singulière. Elle fait face à des défıs spécifiques et sans précédent à l'horizon 2030-2050. Selon les projections des Nations Unies actualisées en 2015, sa population doublera d'ici à 2050 pour atteindre 2,2 milliards d'habitants dont la moitié aura moins de 25 ans. Cette poussée démographique concerne aussi les zones rurales, qui passeront de 620 millions à plus d'un milliard d'habitants dans la même période. La transition démographique est ainsi retardée au-delà de 2050, de même que le dividende démographique - hausse de la productivité économique associée au ralentissement démographique et à la baisse du nombre de personnes à charge.

Autre spécificité : l'agriculture. Elle concentre plus de $60 \%$ de la population active et les exploitations agricoles sont essentiellement familiales, de petite taille et très peu capitalistiques. De ce fait, la transformation structurelle et économique de l'Afrique subsaharienne ne suit pas la trajectoire des pays industrialisés ou émergents. Dans ces pays, l'industrialisation a été facilitée par la croissance rapide de la productivité agricole et le transfert massif des travailleurs agricoles vers les secteurs dits modernes. En Afrique subsaharienne, les secteurs secondaires et tertiaires restent informels et peinent à créer emplois et richesses.

Face à ces défis, les politiques publiques centralisées et sectorielles perdurent malgré les critiques récurrentes à l'encontre de politiques trop distantes, segmentées et fragmentées : force est de constater leur permanence et leur reproduction. Le bilan mitigé de la décentralisation est emblématique de ces rigidités. Les institutions publiques issues de la décentralisation manquent de moyens; elles peinent à trouver leur place dans les organisations politiques et administratives actuelles. Les villes secondaires, défavorisées en termes d'infrastructures et de capacités stratégiques, ne jouent pas leurs rôles de pôle de développement et d'accélérateur du changement structurel. De ce fait, l'industrialisation se concentre dans les périmètres des capitales et des rares grandes agglomérations.

Au Mali, la région de l'Offıce du Niger illustre bien cette tendance. Avec 120000 hectares irrigués et cultivés et $40 \%$ de la production nationale de riz, l'Office du Niger est une région stratégique. Mais le manque d'infrastructures et d'énergie l'empêche de créer une agro-industrie de transformation. Les industries se sont installées à Bamako, à plus 
de 250 kilomètres. Le décollage économique de la région et l'emploi attendent toujours.

Avec toutes ces contraintes, répliquer la trajectoire vécue par les pays industrialisés ou émergents est difficile à envisager. La situation de l'Afrique subsaharienne invite donc à réexaminer les politiques publiques et à s'orienter vers des trajectoires alternatives, avec une attention particulière à la création d'emploi, et dans lesquelles l'agriculture jouera un rôle différent et déterminant.

Mettre l'emploi au cœur de nouvelles stratégies de développement nécessite des approches intersectorielles attentives au travail agricole et à la pluriactivité. Cela implique de favoriser certains modèles de production agricole et de tenir compte de l'emploi engendré par les systèmes alimentaires associés à ces modèles. Cela implique aussi de maximiser l'emploi le long des chaînes de valeur, notamment via la transformation des produits et l'industrie agroalimentaire. Dans le même temps, tous les gisements d'emploi du secteur tertiaire doivent être valorisés. Enfın, ces choix de développement devront considérer la diversité des contextes et aboutir à des solutions adaptées à chaque situation, nationale et locale.

Pour accompagner cette production de politiques innovantes, nous proposons une approche opérationnelle de prospective territoriale, qui amène les acteurs locaux à s'approprier leur avenir et à repenser leur présent. Cette approche a été conçue et testée dans deux régions administratives, l'une au Mali et l'autre à Madagascar (encadré ci-contre).

\section{La prospective territoriale participative : s'approprier le futur pour repenser les dynamiques locales}

La prospective territoriale participative présentée ici est une démarche opérationnelle d'accompagnement des acteurs locaux à l'échelle d'une région administrative, premier niveau de gouvernance après l'échelle nationale. Elle requiert 6 à 18 mois de travail selon la taille de la région, l'accessibilité des données et les connaissances existantes.

Elle combine quatre phases interdépendantes, menées par une équipe de techniciens du développement et de chercheurs en sciences sociales avec l'appui de la société civile locale et de l'administration territoriale déconcentrée et décentralisée.

Un diagnostic rétrospectif. II recense et traite les données disponibles pour la région issues des grandes enquêtes nationales les plus récentes. II utilise aussi les schémas d'aménagement régionaux, les études sectorielles des branches professionnelles, les rapports d'étudiants, les travaux de recherche et d'expertise.

Deux enquêtes. Elles complètent le diagnostic et mettent en lumière les représentations de l'avenir qu'ont les personnes interrogées. Elles concernent, d'une part, au moins 30 personnes ressources choisies à l'issue de la phase de diagnostic et, d'autre part, un échantillon représentatif de la diversité locale d'au moins 200 ménages choisis à dire d'experts et à partir des résultats des grandes enquêtes statistiques disponibles.
La prospective territoriale participative appliquée à deux régions au Mali et à Madagascar

La méthode a été expérimentée dans la région de Ségou au Mali [62500 km², 2,8 millions d'habitants en 2015) et dans le Vakinankaratra au centre de Madagascar $\left[19000 \mathrm{~km}^{2}\right.$, 2 millions d'habitants en 2015). Voici un résumé des quatre phases qui se sont déroulées en 2015.

Diagnostic : rurbanisation, mobilité, poids de l'informel et de l'agriculture. Dans les deux régions, les villages sont de plus en plus nombreux et peuplés, et les villes ne deviennent pas des pôles économiques suffisants pour absorber et sortir de l'agriculture une part significative des nouveaux actifs. La mobilité, saisonnière et de courte distance, est essentielle pour gagner sa vie. L'économie repose sur l'informel (97\% des emplois recensés] et sur la pluriactivité. Mais c'est bien l'agriculture familiale qui domine, puis, loin derrière, les petits métiers urbains.

Enquêtes auprès des habitants : sécurité et gouvernance déficientes. Sont soulignés le besoin de sécurité des biens et des personnes, le manque de dotation en biens publics et les difficultés de la mise en œuvre de la décentralisation. La famille nombreuse, avec au moins quatre enfants par femme, est perçue comme la meilleure assurance face à l'incertitude. L'agriculture concentre l'attention. L'agroalimentaire est la principale perspective d'industrialisation et de croissance, bien plus que l'artisanat, qui est maintenu dans l'informel.

Projections : boum démographique concrétisé à l'échelle régionale. De 2015 à 2035, Ségou passera de 2,5 à 4,2 millions d'habitants et le Vakinankaratra de 2 à 3,1 millions. D'ici à 2035, un million d'emplois devront être créés à Ségou et 700000 dans le Vakinankaratra, soit un rythme de création double de celui des années 2000. Les besoins en éducation et santé sont immenses alors que les moyens n'augmentent que faiblement. Les enjeux d'aménagement du territoire sont cruciaux car de fortes tensions existent déjà entre foncier agricole et ressources naturelles.

Ateliers participatifs : s'approprier le futur pour repenser le présent. Pour les participants, le territoire est multidimensionnel et la sécurité, l'accès à l'énergie et la gouvernance sont essentiels. Huit forces motrices du futur sont mises en avant pour dessiner ensuite des scénarios du futur :

$>$ à Ségou : sécurité, énergie, potentiel minier, infrastructures de communication, secteur informel, orientation du développement économique local, gouvernance territoriale, financement public ;

> dans le Vakinankaratra : sécurité, énergie, pression démographique, capacité d'organisation économique, financement du développement local, inégalités structurelles, mise en marché, pauvreté.

Les participants relèvent que sans politique volontariste et d'ampleur aux échelles locale et nationale, les ressources locales, perçues comme nombreuses, ne seront pas activées. 
Des projections démographiques à 2035 et 2050. Elles caractérisent le défi démographique régional et son impact sur les besoins d'emploi, de foncier agricole, d'éducation et de santé. Ces dynamiques régionales sont comparées aux évolutions nationales afin de comprendre les interrelations entre les échelles spatiales et administratives. Ces projections reposent sur les structures par âge de la population et sur les hypothèses de fécondité et de mortalité construites avec les données du recensement le plus récent et avec celles des Nations Unies.

Un ou plusieurs ateliers participatifs d'au moins une semaine. Ils ont pour but de cerner les forces motrices du territoire et d'élaborer des scénarios du futur. Ils regroupent une trentaine d'acteurs reconnus pour leur connaissance du territoire ou leur capacité à porter la parole de groupes sociaux significatifs. Ces participants sont choisis aussi de manière à couvrir les thématiques clés de la région. Les techniciens de développement et les chercheurs animent l'atelier en s'appuyant sur le diagnostic et sur les projections réalisées. Les participants identifient les forces motrices du territoire, c'est-à-dire celles influençant le plus le futur. Pour cela, les animateurs font discuter ces participants sur les influences d'une cinquantaine de variables prises deux à deux et issues du diagnostic. Il leur est demandé d'être attentifs aux processus déjà à l'œuvre et aux processus latents pouvant être moteurs dans l'avenir. Ensuite, des scénarios exploratoires cohérents sont élaborés en combinant les différents états plausibles des forces motrices. Les participants passent ainsi d'un ensemble de représentations individuelles à une appropriation collective des enjeux.

En se projetant de cette façon dans l'avenir et en s'appropriant les futurs possibles, les décideurs locaux peuvent réinterpréter le présent et proposer des changements dans les politiques actuelles. Le travail collectif des ateliers et les échanges qui en résultent au-delà de ce cercle aboutissent à une connaissance partagée et multisectorielle du territoire. Les facteurs d'évolution et les marges d'action sont également mieux appréhendés.

\section{Mettre le territoire et ses acteurs au cœur des politiques publiques}

Cette démarche de prospective territoriale est innovante parce qu'elle constitue un socle pour transformer les politiques de développement d'un territoire. La suite du travail consisterait à repartir des scénarios et des forces motrices pour élaborer collectivement, entre acteurs compétents, des actions qui tendent vers les futurs désirables. Mais même sans cette suite, les acteurs locaux, parce qu'ils participent au diagnostic et aux scénarios, ont une plus grande conscience de l'ampleur des défis à l'échelle de leur territoire et de leur capacité à y répondre. Or ces défis sont d'ordinaire présentés comme relevant des échelles nationale et globale auxquelles ils ne sont pas conviés à intervenir.

Les deux prospectives territoriales menées au Mali et à Madagascar font ressortir l'agriculture comme une clé majeure du futur. Elles montrent que des modèles agricoles porteurs d'emplois et moteurs de développement multisectoriel ne peuvent être construits qu'à certaines conditions. En particulier, ces nouveaux modèles doivent maximiser un ensemble de services environnementaux et sociaux en plus du volume de production agricole. Une agriculture familiale intensive en travail et fondée sur l'agroécologie pourrait accroître les performances écologiques, économiques et sociales des systèmes agraires et alimentaires. Cette option mériterait d'être explorée en tenant compte des situations locales et de leurs perspectives différenciées d'emploi et de diversification économique. II conviendrait aussi d'en évaluer les performances par rapport à d'autres options, comme l'agrobusiness et les modèles productivistes de type "révolution verte » promus de fait par les politiques agricoles de nombreux pays d'Afrique subsaharienne.

Également, les deux prospectives mettent en évidence quatre actions essentielles pour relever les défis actuels des zones rurales africaines:

> identifier et renforcer les compétences, savoir-faire et bonnes pratiques de l'administration des régions et plus largement des services déconcentrés de l'État ;

$>$ renforcer les liens entre l'agriculture et les activités présentes en amont et en aval ;

$>$ renforcer les liens entre la capitale, les villes secondaires et les territoires ruraux;

$>$ rééquilibrer les services et les infrastructures entre les villages, les villes secondaires et la capitale pour rendre possible la diversification des activités et des emplois.

Pour réaliser et démultiplier ces actions, les politiques de décentralisation doivent être renforcées et adaptées, en particulier celles qui partagent la décision stratégique entre les échelles nationale et locale. La décentralisation n'est en effet effective que si des compétences locales existent, mais elle nécessite une organisation nationale pour la relayer. C'est donc aussi une question de volonté gouvernementale de voir s'exercer localement des compétences qui étaient auparavant celle de l'État. Rendre la décentralisation active donne aux décideurs et politiques locaux la capacité à anticiper et à planifier le développement territorial.

Ainsi, avec cette méthode de prospective territoriale, les décideurs locaux disposent d'un instrument projetant les enjeux à l'horizon de la prochaine génération. S'ils ne peuvent pas prévoir le futur, ils peuvent utiliser les scénarios pour apprécier les trajectoires actuelles et pour imaginer des politiques publiques territoriales innovantes et soutenables. Cette méthode peut permettre de passer de la projection du territoire à la définition d'un projet de territoire viable et partagé. En faisant varier l'état d'une force motrice qui influe sur le futur, il est en effet possible d'anticiper les impacts de ce changement sur la trajectoire locale, à condition de tenir compte aussi des influences externes, des enseignements du diagnostic territorial et des besoins mesurés en capitaux et en infrastructures. De la même façon, il est possible de se servir des images du futur ainsi construites pour apprécier l'impact des politiques publiques que l'on mettrait en œuvre maintenant. 
Ce Perspective $\mathrm{n}^{\circ} 44$ est issu des travaux de l'expertise Prospective territoriale sur les dynamiques démographiques et le développement rural en Afrique subsaharienne et à Madagascar financée par l'Agence française de développement [Afd, www.afd.fr] et réalisée en 2015-2016 par le Cirad en association avec l'Institut d'économie rurale du Mali [IER] et l'Institut international de sciences sociales à Madagascar [IISS, https://iissmada.wordpress.com/]. L'approche choisie, à visée prospective, a combiné diagnostic territorial, étude démographique et participation des acteurs du territoire.

Cette expertise est publiée dans la synthèse suivante :

Sourisseau J.-M., Bélières J.-F., Bourgeois R., Soumaré M., Rasolofo P., Guengant J.-P., Bougnoux N., 2017. Penser ensemble l'avenir d'un territoire. Diagnostic et prospective territoriale au Mali et à Madagascar. Paris, Afd. Études de l'Afd n 15, 179 p. ISSN 2492-8313. www.afd.fr/fr/penser-ensemble-avenir-territoire.

Ces travaux ont donné lieu à d'autres publications :

Bourgeois R., Sourisseau J.-M., Rasolofo P., Bougnoux N., Ramanitriniony Haja K., Rabeandriamaro H., Bélières J.-F., 2016. Atelier de prospective participative Les avenirs de Vakinankaratra en 2035. Paris, Afd, 38 p.

http://agritrop.cirad.fr/580520/.
Bourgeois R., Sourisseau J.-M., Soumaré M., Coulibaly B., Bélières J.-F., Bougnoux N., 2016. Atelier de prospective participative Les avenirs de Ségou en 2035. Paris, Afd, 35 p. http://agritrop.cirad.fr/580519/.

Sourisseau J.-M., Bougnoux N., Bélières J.-F., Bourgeois R., 2017. Faire et penser " territoire » en envisageant le futur : prospective territoriale dans les régions de Ségou au Mali et du Vakinankaratra à Madagascar. In Des territoires vivants pour transformer le monde. Caron P., Valette E., Wassenaar T., Coppens D'Eeckenbrugge G., Papazian V. [Eds]. Versailles, Editions Quae, p. 251-261. ISBN 978-2-7592-2654-2.

Sourisseau J.-M., Rasolofo P., Bélières J.-F., Guengant J.-P., Ramanitriniony Haja K., Bourgeois R., Razafimiarantsoa Tovonirina T., Andrianantoandro Voahirana T., Ramarijaona M., Burnod P., Rabeandriamaro H., Bougnoux N., 2016. Diagnostic territorial de la région du Vakinankaratra à Madagascar. Paris, Afd, 157 p. http://agritrop.cirad.fr/580518/.

Sourisseau J.-M., Soumaré M., Bélières J.-F., Guengant J.-P., Bourgeois R., Coulibaly B., Traoré S., 2016. Diagnostic territorial de la région de Ségou au Mali. Paris, Afd, 150 p. http://agritrop.cirad.fr/580517/.

\section{Quelques mots sur...}

Jean-Michel Sourisseau est socio-économiste à I'UMR ART-Dev au Cirad (Acteurs, ressources et territoires dans le développement, http://art-dev.cnrs.fr]. Il est le point focal du Cirad sur la thématique de l'agriculture familiale.

jean-michel.sourisseau@cirad.fr

Nathalie Bougnoux est responsable d'équipe projet au sein de la division agriculture, développement rural et biodiversité à l'Afd (Agence française de développement, www.afd.fr]. Elle a longtemps travaillé sur les dynamiques démographiques et migratoires et a initié plusieurs travaux de prospective à l'Afd.

bougnouxn@afd.fr

Jean-François Bélières est agroéconomiste à l'UMR ART-Dev au Cirad en poste à Madagascar au Fofifa (Centre national de la recherche appliquée au développement rural, Madagascar, www.fofifa.mg) dans le dispositif de recherche et d'enseignement en partenariat du Cirad SPAD [Systèmes de production d'altitude et durabilité à Madagascar, www.dp-spad.org]. II travaille sur les effets des politiques de développement rural sur les exploitations agricoles et les économies locales. jean-francois.belieres@cirad.fr

Robin Bourgeois est chercheur prospectiviste à I'UMR ART-Dev au Cirad. Actuellement en Afrique du Sud, il conduit des recherches appliquées en matière de prospective, gouvernance et développement territorial. robin.bourgeois@cirad.fr

Mamy Soumarē est géographe, chercheur à l'Institut d'économie rurale au Mali (IER) et professeur à I'Université de Bamako. Ses recherchent portent sur la représentation et la modélisation des dynamiques spatiales.

soumare_mamy@hotmail.com

Patrick Rasolofo est économiste en développement rural. II est fondateur et directeur de l'Institut international des sciences sociales (IISS, Madagascar, https://iissmada.wordpress.com/]

iiss@moov.mg

(C) (1) $\begin{aligned} & \text { Cette œuvre est mise à dis- } \\ & \text { position selon les termes de } \\ & \text { la Licence Creative Com- }\end{aligned}$
mons CC-BY-NC-SA 4.0 : Attribution - Pas
d'Utilisation Commerciale - Partage dans les
Mêmes Conditions - 4.0 International http://
creativecommons.org/licenses/by-nc-sa/4.0/
deed.fr

Pour citer ce document

Sourisseau J.-M., Bougnoux N., Bélières J.-F Bourgeois R., Soumaré M., Rasolofo P., 2017. Imaginer l'avenir des territoires pour relever les défis de la démographie et de l'emploi en Afrique subsaharienne. Cirad, Montpellier, Perspective 44.

https://doi.org/10.19182/agritrop/00002

\section{Quelques liens}

Fao, 2017. La situation mondiale de l'alimentation et de l'agriculture. Mettre les systèmes alimentaires au service d'une transformation rurale inclusive. Fao, Rome. ISBN 978-92-5-209873-7. www.fao.org/state-of-food-agriculture/fr/.

Losch B., 2016. Structural transformation to boost youth labour demand in sub-Saharan Africa: The role of agriculture, rural areas and territorial development. Geneva, International Labour Office (ILO), Employment working paper $n^{\circ} 204$. Www.ilo.org/employment/Whatwedo/Publications/working-papers/WCMS_533993/lang--en/index.htm.

Pesche D., Losch B., Imbernon J. (eds.), 2016. Une nouvelle ruralité émergente - Regards croisés sur les transformations rurales africaines. Seconde édition revue et augmentée, Nepad et Cirad, 76 p. ISBN 978-2-87614-718-8. www.cirad.fr/publications-ressources/edition/ etudes-et-documents/atlas-une-nouvelle-ruralite-emergente-enafrique-2e-edition.

United Nations, Department of Economic and Social Affairs, Population Findings and Advance Tables. Working Paper ESA/P/WP/248. https:// esa.un.org/unpd/wpp/.

\section{Agence française de développement (Afd)

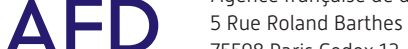 75598 Paris Cedex 12 • France www.afd.fr} Division, 2017. World Population Prospects: The 2017 Revision, Key
Directeur de la publication: Michel Eddi, Rédacteur.e.s en chef : Patrick Caron, direction générale déléguée à la recherche et à la stratégie : Cécile Fovet-Rabot, délégation à l'information scientifique et technique Président directeur général du Cirad

Mise en pages et illustrations : Delphine Lavastre-Guard, délégation à la communication Diffusion : Christiane Jacquet, délégation à la communication Courriel : perspective@cirad.fr www.cirad.fr/publications-ressources/edition/perspective-policy-brief perspactive ISSN-L 2275-9131 